\title{
SUMMARY OF THE FIRST EIGHT YEARS OF THE PRAIRIE NEST RECORDS SCHEME
}

\author{
by Robert R. Taylor, University of Saskatchewan, Regina
}

The Prairie Nest Records Scheme, to the end of its eighth year of operation, has accumulated a total of 7,221 record cards representing 236 species of birds. The records included in the files of the scheme cover the province of Manitoba, Saskatchewan, Alberta, and the Northwest Territories.

Since its inception in 1958, the Prairie Nest Records Scheme has enjoyed the support of more than 100 contributors, mostly non-professional.

Many of the cards contributed to the scheme record the presence of more than one nest in a particular location. For example, cards representing certain colony nesting birds such as gulls and terns may contain up to 20 nests. In the case of some waterfowl species and some species of upland game birds, the cards represent broods that have left the nest, rather than the nest itself.

During the eight years of operation of the scheme, a number of biologists have requested information from the files for use in scientific studies in which they were engaged. It is hoped that by presenting the following listing of cards available for each species, more extensive use of the information contained on the Prairie Nest Record Cards will be encouraged.

Summary of Cards in Prairie Nest Records Scheme Files to end of 1965

Common Loon, 24; Arctic Loon, 2; Red - throated Loon, 7; Red-necked Grebe, 3; Horned Grebe, 43; Eared Grebe, 39; Western Grebe 7; Piedbilled Grebe, 38; White Pelican, 5; Double-crested Cormorant, 13; Great Blue Heron, 14; Black-crowned Night Heron, 5; American Bittern, 11; Mute Swan, 2; Whistling Swan, 13; Canada Goose, 36; White-fronted Goose, 30; Mallard, 301; Gadwall, 6; Pintail, 87; Green-winged Teal, 15; Blue-winged Teal, 73; American Widgeon, 29; Shoveler, 16; Redhead, 19; Ringnecked Duck, 3; Canvasback, 46; Greater Scaup, 6; Lesser Scaup, 26; Common Goldeneye, 7; Barrow's Goldeneye, 3; Bufflehead, 8; Old Squaw, 18; King Eider, 1; White-winged Scoter, 22; Ruddy Duck 26; Common Merganser, 3; Red-breasted Merganser, 14; Turkey Vulture, 1; Goshawk, 1; Sharp-shinned Hawk, 11; Cooper's Hawk, 35; Red-tailed Hawk, 136;

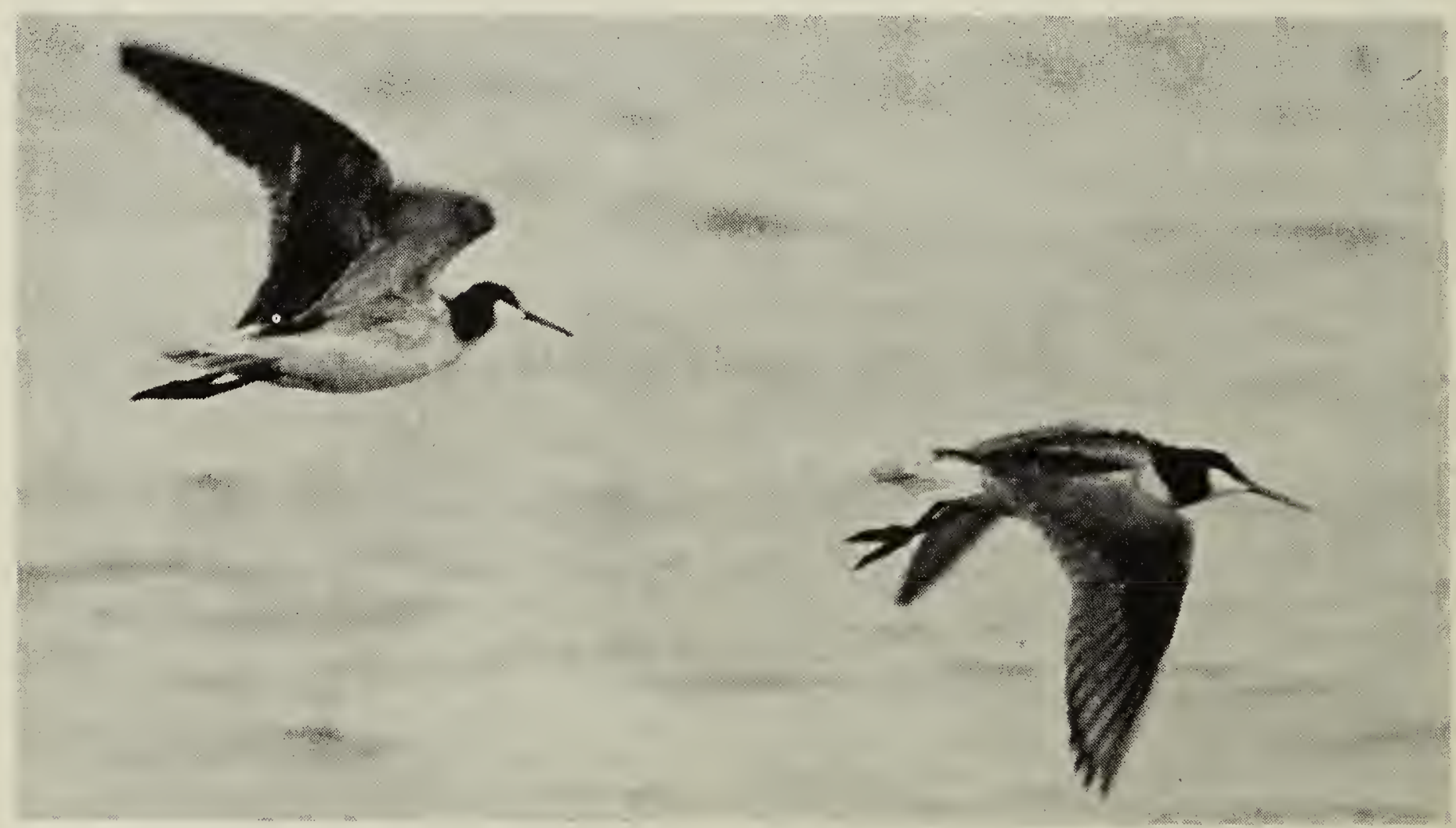


Broad-winged Hawk, 4; Swainson's Hawk, 40; Rough-legged Hawk, 26; Ferruginous Hawk, 29; Golden Eagle, 7; Bald Eagle, 14; Marsh Hawk, 71; Osprey, 3; Gyrfalcon, 7; Prairie Falcon, 35; Peregrine Falcon, 17; Pigeon Hawk, 32; Sparrow Hawk, 34; Blue Grouse, 1; Spruce Grouse, 7; Ruffed Grouse, 25; Willow Ptarmigan, 32; Rock Ptarmigan, 1; White - tailed Ptarmigan, 1; Sharp-tailed Grouse, 17; Sage Grouse, 2; Ring-necked Pheasant, 2; Gray Partridge, 13; Whooping Crane, 4; Sandhill Crane, 2; Virginia Rail, 8; Sora, 29; American Coot, 245; Semipalmated Plover, 20; Piping Plover, 5; Killdeer, 167; Golden Plover, 3; Black-bellied Plover, 1; Common Snipe, 14; Long-billed Curlew 3; Upland Plover, 6; Spotted Sandpiper, 28; Solitary Sandpiper, 3; Willet, 15; Greater Yellowlegs, 3; Lesser Yellowlegs, 3; Pectoral Sandpiper, 1; White-rumped Sandpiper, 2; Baird's Sandpiper, 4; Least Sandpiper, 1; Stilt Sandpiper, 1; Semipalmated Sandpiper, 5; Marbled Godwit, 15; American Avocet, 39; Red Phalarope, 1; Wilson's Phalarope, 35; Northern Phalarope, 7; Parasitic Jaeger, 3; Long-tailed Jaeger, 5; Glaucous Gull, 2; Herring Gull, 36; California Gull, 10; Ring-billed Gull, 12; Mew Gull, 6; Franklin's Gull, 5; Bonaparte's Gull, 2; Forster's Tern, 4; Common Tern, 42; Arctic Tern, 43; Black Tern, 63; Rock Dove, 4; Mourning Dove, 188; Black-billed Cuckoo, 14; Screech Owl, 2; Great Horned Owl, 141; Snowy Owl, 1; Hawk Owl, 2; Burrowing Owl, 6; Barred Owl, 1; Long-eared Owl, 57; Short-eared Owl, 31; Boreal Owl, 1; Saw-whet Owl, 2; Common Nighthawk, 15; Rubythroated Hummingbird, 1; Rufous Hummingbird, 3; Belted Kingfisher, 10; Yellow-shafted Flicker, 78; Redshafted Flicker, 3; Pileated Woodpecker, 2; Red-headed Woodpecker, 2; Yellow-bellied Sapsucker, 25; Hairy Woodpecker, 7; Downy Woodpecker, 17; Eastern Kingbird, 129; Western Kingbird, 52; Great Crested Flycatcher, 6; Eastern Phoebe, 87; Say's Phoebe, 11; Traill's Flycatcher, 3; Least Flycatcher, 61; Dusky Flycatcher, 3; Western Wood Pewee, 11;
Horned Lark, 107; Violet-Green Swallow, 1; Tree Swallow, 115; Bank Swallow, 31; Rough-winged Swallow, 9; Barn Swallow, 282; Cliff Swallow, 43; Purple Martin, 14; Gray Jay, 1; Blue Jay, 4; Black-billed Magpie, 172; Common Raven, 5; Common Crow, 127; Black-capped Chickadee, 8; White-breasted Nuthatch, 2; Dipper, 1; House Wren, 152; Long-billed Marsh Wren, 20; Short-billed Marsh Wren, 16; Rock Wren, 1; Mockingbird, 1 ; Catbird, 102 ; Brown Thrasher, 40; Robin, 353; Hermit Thrush, 4; Swainson's Thrush, 7; Gray-cheeked Thrush, 14; Veery, 18; Eastern Bluebird, 8; Mountain Bluebird, 42; Townsend's Solitaire, 1; Ruby - crowned Kinglet, 2; Water Pipit, 6; Sprague's Pipit, 4; B oh em ian Waxwing, 7; Cedar Waxwing, 109; Loggerhead Shrike, 28; Starling, 19; Solitary Vireo, 1; Red-eyed Vireo, 14; Philadelphia Vireo, 1; Warbling Vireo, 25; Black-and-white Warbler, 2; Tennessee Warbler, 1 ; Orange-crowned Warbler, 1; Yellow Warbler, 100; Magnolia Warbler, 1; Myrtle Warbler, 6; Audubon's Warbler, 2; Chestnut-sided Warbler, 1; Blackpoll Warbler, 1; Palm Warbler, 2; Ovenbird, 4; Yellowthroat, 5; Yellow-breasted Chat, 2; American Redstart, 6; House Sparrow, 41; B obolink, 15; Western Meadowlark, 67; Yellow-headed Blackbird, 102; Red-winged Blackbird, 410; Baltimore Oriole, 29; Rusty Blackbird, 3; Brewer's Blackbird, 163; Common Grackle, 59; Rose-breasted Grosbeak, 9; Purple Finch, 5; Pine Grosbeak, 1; Hoary Redpoll, 17; Common Redpoll, 17; Pine Siskin, 4; American Goldfinch, 23; Rufous-sided Towhee, 2; Lark Bunting, 32; Grasshopper Sparrow, 1 ; Le Conte's Sparrow, 2 : Sharptailed Sparrow, 2; Vesper Sparrow, 111; Lark Sparrow, 7; Slate-colored Junco, 11; Oregon Junco, 3; Tree Sparrow, 33; Chipping Sparrow, 51; Clay-colored Sparrow, 173; Harris' Sparrow, 18; White-crowned Sparrow, 19; White-throated Sparrow, 7; Fox Sparrow, 1; Lincoln's Sparrow, 4; Swamp Sparrow, 2; Song Sparrow, 81; McCowan's Longspur, 3; Lapland Longspur, 23; Chestnut-collared Longspur 112; Snow Bunting, 1. 